\title{
Plasmonic-organic hybrid (POH) modulators for OOK and BPSK signaling at $40 \mathrm{Gbit} / \mathrm{s}$
}

\author{
A. Melikyan, ${ }^{1, *}$ K. Koehnle, ${ }^{1}$ M. Lauermann, ${ }^{2}$ R. Palmer, ${ }^{2}$ S. Koeber, ${ }^{2}$ S. Muehlbrandt, ${ }^{1}$ \\ P. C. Schindler, ${ }^{2}$ D. L. Elder, ${ }^{3}$ S. Wolf, ${ }^{2}$ W. Heni, ${ }^{4}$ C. Haffner, ${ }^{4}$ Y. Fedoryshyn, ${ }^{4}$ D. \\ Hillerkuss, ${ }^{4}$ M. Sommer, ${ }^{1}$ L. R. Dalton, ${ }^{3}$ D. Van Thourhout, ${ }^{5}$ W. Freude, ${ }^{2}$ M. Kohl, ${ }^{1}$ J. \\ Leuthold $^{4,6}$ and C. Koos ${ }^{1,2,7}$ \\ ${ }^{1}$ Institute of Microstructure Technology (IMT), Karlsruhe Institute of Technology (KIT), Karlsruhe, Germany \\ ${ }^{2}$ Institute of Photonics and Quantum Electronics (IPQ), Karlsruhe Institute of Technology (KIT), Karlsruhe, \\ Germany \\ ${ }^{3}$ University of Washington, Department of Chemistry, Seattle, USA \\ ${ }^{4}$ Swiss Federal Institute of Technology (ETH), 8092 Zurich, Switzerland \\ ${ }^{5}$ Photonics Research Group, Ghent University - IMEC, Gent, Belgium \\ juergleuthold@ethz.ch \\ 7christian.koos@kit.edu \\ *argishti.melikyan@kit.edu
}

\begin{abstract}
We report on high-speed plasmonic-organic hybrid MachZehnder modulators comprising ultra-compact phase shifters with lengths as small as $19 \mu \mathrm{m}$. Choosing an optimum phase shifter length of $29 \mu \mathrm{m}$, we demonstrate $40 \mathrm{Gbit} / \mathrm{s}$ on-off keying (OOK) modulation with direct detection and a BER $<6 \times 10^{-4}$. Furthermore, we report on a $29 \mu \mathrm{m}$ long binary-phase shift keying (BPSK) modulator and show that it operates error-free $\left(\mathrm{BER}<1 \times 10^{-10}\right)$ at data rates up to $40 \mathrm{Gbit} / \mathrm{s}$ and with an energy consumption of $70 \mathrm{fJ} / \mathrm{bit}$.
\end{abstract}

(C)2015 Optical Society of America

OCIS codes: (250.5403) Plasmonics; (240.6680) Surface plasmons; (250.4110) Modulators.

\section{References and links}

1. D. Miller, "Device requirements for optical interconnects to silicon chips," Proc. IEEE 97(7), 1166-1185 (2009).

2. G. T. Reed, G. Mashanovich, F. Y. Gardes, and D. J. Thomson, "Silicon optical modulators," Nat. Photonics 4(8), 518-526 (2010).

3. D. M. Gill, M. Rasras, K.-Y. Tu, Y.-K. Chen, A. E. White, S. S. Patel, D. Carothers, A. Pomerene, R. Kamocsai, C. Hill, and J. Beattie, "Internal bandwidth equalization in a CMOS-compatible Si-ring modulator," IEEE Photon. Technol. Lett. 21(4), 200-202 (2009).

4. M. R. Watts, D. C. Trotter, R. W. Young, and A. L. Lentine, "Ultralow power silicon microdisk modulators and switches," in IEEE 5th International Conference on Group IV Photonics (GFP, 2008), paper WA2.

5. P. Dong, W. Qian, H. Liang, R. Shafiiha, D. Feng, G. Li, J. E. Cunningham, A. V. Krishnamoorthy, and M. Asghari, "Thermally tunable silicon racetrack resonators with ultralow tuning power," Opt. Express 18(19), 20298-20304 (2010).

6. L. Liao, A. Liu, J. Basak, H. Nguyen, R. Cohen, N. Izhaky, and M. Paniccia, "40 Gbit s-1 silicon optical modulator for highspeed applications,” Electron. Lett. 43(22), 1196-1197 (2007).

7. E. Timurdogan, C. M. Sorace-Agaskar, J. Sun, E. Shah Hosseini, A. Biberman, and M. R. Watts, "An ultralow power athermal silicon modulator," Nat. Commun. 5, 4008 (2014).

8. H. C. Nguyen, S. Hashimoto, M. Shinkawa, and T. Baba, "Compact and fast photonic crystal silicon optical modulators," Opt. Express 20(20), 22465-22474 (2012).

9. J. Leuthold, C. Koos, W. Freude, L. Alloatti, R. Palmer, D. Korn, J. Pfeifle, M. Lauermann, R. Dinu, S. Wehrli, M. Jazbinsek, G. Peter, M. Waldow, T. Wahlbrink, J. Bolten, H. Kurz, M. Fournier, J. Fedeli, H. Yu, and W. Bogaerts, "Silicon-organic hybrid electro-optical devices," IEEE J. Sel. Top. Quantum Electron. 19(6), 3401413 (2013).

10. J. M. Brosi, C. Koos, L. C. Andreani, M. Waldow, J. Leuthold, and W. Freude, "High-speed low-voltage electrooptic modulator with a polymer-infiltrated silicon photonic crystal waveguide," Opt. Express 16(6), 4177-4191 (2008).

11. J. Li, T. P. White, L. O'Faolain, A. Gomez-Iglesias, and T. F. Krauss, "Systematic design of flat band slow light in photonic crystal waveguides," Opt. Express 16(9), 6227-6232 (2008).

12. P. Dong, C. Xie, L. Chen, N. K. Fontaine, and Y. K. Chen, "Experimental demonstration of microring quadrature phase-shift keying modulators," Opt. Lett. 37(7), 1178-1180 (2012). 
13. J. A. Dionne, K. Diest, L. A. Sweatlock, and H. A. Atwater, "PlasMOStor: A metal-oxide-Si field effect plasmonic modulator," Nano Lett. 9(2), 897-902 (2009).

14. A. Melikyan, N. Lindenmann, S. Walheim, P. M. Leufke, S. Ulrich, J. Ye, P. Vincze, H. Hahn, T. Schimmel, C. Koos, W. Freude, and J. Leuthold, "Surface plasmon polariton absorption modulator," Opt. Express 19(9), 88558869 (2011).

15. V. J. Sorger, N. D. Lanzillotti-Kimura, R.-M. Ma, and X. Zhang, "Ultra-compact silicon nanophotonic modulator with broadband response," J. Nanophotonics 1(1), 17-22 (2012).

16. W. Cai, J. S. White, and M. L. Brongersma, "Compact, high-speed and power-efficient electrooptic plasmonic modulators," Nano Lett. 9(12), 4403-4411 (2009).

17. S. Zhu, G. Q. Lo, and D. L. Kwong, "Theoretical investigation of silicon MOS-type plasmonic slot waveguide based MZI modulators," Opt. Express 18(26), 27802-27819 (2010).

18. V. E. Babicheva and A. V. Lavrinenko, "Plasmonic modulator optimized by patterning of active layer and tuning permittivity," Opt. Commun. 285(24), 5500-5507 (2012).

19. A. Melikyan, L. Alloatti, A. Muslija, D. Hillerkuss, P. C. Schindler, J. Li, R. Palmer, D. Korn, S. Muehlbrandt, D. Van Thourhout, B. Chen, R. Dinu, M. Sommer, C. Koos, M. Kohl, W. Freude, and J. Leuthold, "High-speed plasmonic phase modulators," Nat. Photonics 8(3), 229-233 (2014).

20. S. Papaioannou, D. Kalavrouziotis, K. Vyrsokinos, J.-C. Weeber, K. Hassan, L. Markey, A. Dereux, A. Kumar, S. I. Bozhevolnyi, M. Baus, T. Tekin, D. Apostolopoulos, H. Avramopoulos, and N. Pleros, "Active plasmonics in WDM traffic switching applications," Sci Rep 2(652), 652 (2012).

21. C. Haffner, W. Heni, Y. Fedoryshyn, D. Elder, A. Melikyan, B. Baeuerle, J. Niegemann, A. Emboras, A. Josten, F. Ducry, M. Kohl, L. Dalton, D. Hillerkuss, C. Hafner, and J. Leuthold, "High-speed plasmonic Mach-Zehnder modulator in a waveguide," in European Conference on Optical Communications (ECOC, 2014), paper PD2.6.

22. J. Lee, N. Kaneda, T. Pfau, A. Konczykowska, F. Jorge, J.-Y. Dupuy, and Y.-K. Chen, "Serial 103. 125 Gbit/s transmission over $1 \mathrm{~km}$ SSMF for low- cost, short-reach optical interconnects," in Optical Fiber Communication Conference (OFC, 2014), paper Th5A.5.

23. D. F. P. Pile and D. K. Gramotnev, “Adiabatic and nonadiabatic nanofocusing of plasmons by tapered gap plasmon waveguides,” Appl. Phys. Lett. 89(4), 041111 (2006).

24. R. Palmer, S. Koeber, D. L. Elder, M. Woessner, W. Heni, D. Korn, M. Lauermann, W. Bogaerts, L. Dalton, W. Freude, J. Leuthold, and C. Koos, "High-speed, low drive-voltage silicon-organic hybrid modulator based on a binary-chromophore electro-optic material," IEEE J. Light. Technol. 32(16), 2726-2734 (2014).

25. R. Schmogrow, B. Nebendahl, M. Winter, A. Josten, D. Hillerkuss, S. Koenig, J. Meyer, M. Dreschmann, M. Huebner, C. Koos, J. Becker, W. Freude, and J. Leuthold, "Error vector magnitude as a performance measure for advanced modulation formats," IEEE Photon. Technol. Lett. 24(1), 61-63 (2012).

26. D. A. B. Miller, "Energy consumption in optical modulators for interconnects," Opt. Express 20(S2 Suppl 2), A293-A308 (2012).

27. M. R. Watts, W. A. Zortman, D. C. Trotter, R. W. Young, and A. L. Lentine, "Low-voltage, compact, depletionmode, silicon Mach-Zehnder modulator," IEEE J. Sel. Top. Quantum Electron. 16(1), 159-164 (2010).

28. F. Chang, K. Onohara, and T. Mizuochi, "Forward error correction for $100 \mathrm{G}$ transport networks," IEEE Commun. Mag. 48(3), 48-55 (2010).

\section{Introduction}

Short and mid-range optical links utilizing wavelength division multiplexed (WDM) demand high-speed and compact integrated light modulators, which operate in a broad optical wavelength range at an energy consumption of a few femtojoules per bit [1]. Compact integrated modulators are commonly realized on the silicon photonic platform using resonant structures such as ring or disk resonators, or as photonic crystals [2-11]. These devices exploit resonant enhancement of the light-matter interaction to enable compact implementations and low driving voltages. As an example, a modulator with a footprint of only $18 \ldots 25 \mu \mathrm{m}^{2}$ has been reported employing microdisk resonators [7]. However, ring or disk modulators require careful matching of the carrier wavelength and of the resonance wavelength, by, e.g., heaters, which comes along with additional power consumption. Moreover, these devices do not open an easy route to advanced modulation formats such as quadrature amplitude modulation (QAM) [12]. As an alternative, photonic crystal (PC) modulators $[8,10,11]$ can be used, allowing lengths of down to $50 \mu \mathrm{m}$ [8]. However, PC based modulators usually occupy larger chip areas than ring-resonator modulators due to the large transverse dimensions of the PC sections. Furthermore, both for ring resonators and photonic crystal modulators, the optical bandwidth is inherently limited by the resonant nature of the device. These limitations can be overcome by plasmonic integration, which enables compact modulators without the need of making the devices resonant [13-20]. 
A high-speed plasmonic phase modulator with a length of $29 \mu \mathrm{m}$ and with large RF and optical bandwidths have recently been reported by our group, exploiting the benefits of plasmonic-organic hybrid $(\mathrm{POH})$ integration [19]. This approach is based on combining plasmonic slot waveguide with electro-optic (EO) organic cladding materials, thereby enabling binary phase-shift keying (BPSK) at $40 \mathrm{Gbit} / \mathrm{s}$. More recently, our group has reported on an ultra-compact plasmonic-organic hybrid (POH) Mach-Zehnder modulator (MZM) generating BPSK signals at data rates of up to $72 \mathrm{Gbit} / \mathrm{s}$ with a bit error ratio (BER) of $3 \times 10^{-3}[21]$.

However, the application of plasmonic modulators within short-reach optical links calls for much lower BERs in order to minimize coding overhead for forward error correction (FEC), high-speed signal processing and the associated power consumption as well as associated latency. Furthermore, on-off-keying (OOK) with direct detection remains the most promising method for short-reach transmissions because of its technical simplicity and low implementation costs [22]. Thus, a plasmonic MZM that can reliably generate on-off keying (OOK) signals is in the focus of interest.

Here, we report on highly efficient POH modulators that enable the lowest BER that has so far been achieved with plasmonic devices at technically relevant data rates. In particular, applying a cladding of state-of-the-art organic material YLD124 / PSLD41 on our $29 \mu \mathrm{m}$-long phase modulator leads to a voltage-length product $\mathrm{V}_{\pi} \times \mathrm{L}$ of $0.45 \mathrm{~V} \mathrm{~mm}$. Using this device, we demonstrate error-free (BER $<1 \times 10^{-10}$ ) binary-phase shift keying (BPSK) signaling at data rates up to $40 \mathrm{Gbit} / \mathrm{s}$. This BER is 5 orders of magnitude smaller than shown by us previously [19]. No FEC is required, and the modulator's energy consumption amounts to 70 $\mathrm{fJ} / \mathrm{bit}$. Furthermore, we report on the generation and direct detection of $40 \mathrm{Gbit} / \mathrm{s}$ OOK signals using plasmonic-organic hybrid ( $\mathrm{POH})$ Mach-Zehnder modulators (MZM). We test different devices featuring footprints of $21 \times 19 \mu \mathrm{m}^{2}, 21 \times 29 \mu \mathrm{m}^{2}$ and $21 \times 39 \mu \mathrm{m}^{2}$. We discuss the optimum length of a POH MZM and find that $29 \mu \mathrm{m}$ long plasmonic phase shifter (PS) sections represents an optimum in the sense that the necessary extinction ratio is achieved at an acceptable modulator insertion loss. This device allows generation of 30 $\mathrm{Gbit} / \mathrm{s}, 35 \mathrm{Gbit} / \mathrm{s}$ and $40 \mathrm{Gbit} / \mathrm{s}$ OOK signals with a BER $<6 \times 10^{-4}$ that is below the threshold of hard-decision FEC codes. Disregarding FEC, the energy consumption of these POH MZMs is in the range of $75 \ldots 225 \mathrm{fJ} /$ bit, depending on the device capacitance which is determined by the length of the PS. To the best of our knowledge, our experiments demonstrate the first error-free generation of a BPSK signal using a plasmonic modulator, and the first example of $40 \mathrm{Gbit} / \mathrm{s}$ OOK signaling using a plasmonic MZM.

\section{Modulator design and fabrication}

We realized our plasmonic modulators on the silicon photonic platform. The devices are based on a silicon-on-insulator wafer having a $2 \mu \mathrm{m}$ thick buried oxide and a silicon device layer with a thickness of $220 \mathrm{~nm}$. Silicon nanowire waveguides with a width of $450 \mathrm{~nm}$ and a height of $220 \mathrm{~nm}$ are used as access waveguides to the phase modulator sections. Light is coupled in and out of the silicon nanowires using silicon grating couplers. The BPSK modulator is constructed of a single high-speed plasmonic phase shifter (PS) operating as a phase modulator (PM), see Fig. 1(a) and 1(b) [19]. Figure 1(a) gives an artist's impression of the POH PM. Mode conversion between the quasi-TE mode of the silicon waveguide and the gap surface plasmon polariton (SPP) is accomplished via a tapered silicon waveguide enclosed by a tapered gap plasmon waveguide [23]. The phase of the optical signal is modulated in the $29 \mu \mathrm{m}$ long plasmonic PM section, labelled as "Phase Modulator (PM)" in Fig. 1(a) and "PM" in Fig. 1(b). The POH MZM for OOK consists of one PS placed in each of the two arms of a Mach-Zehnder interferometer (MZI), which is realized on a silicon planar lightwave circuit (PLC), see Fig. 1(c) and 1(d). The MZI is designed with unbalanced arms to enable adjustment of the operating point by wavelength tuning. Standard silicon photonic multimode interference (MMI) couplers with insertion losses smaller than $0.5 \mathrm{~dB}$

\#226747 - \$15.00 USD Received 12 Nov 2014; revised 12 Mar 2015; accepted 16 Mar 2015; published 10 Apr 2015

(C) 2015 OSA 20 Apr 2015 | Vol. 23, No. 8 | DOI:10.1364/OE.23.009938 | OPTICS EXPRESS 9940 
serve as optical power splitters/combiners. High-speed phase modulation is performed by the PS exploiting the Pockels effect in an electro-optic organic cladding material [19]. Applying a voltage between the metal electrodes changes the refractive index of the electro-optic (EO) material in the slot due to the Pockels effect, and therefore influences the phase velocity of the plasmonic mode.

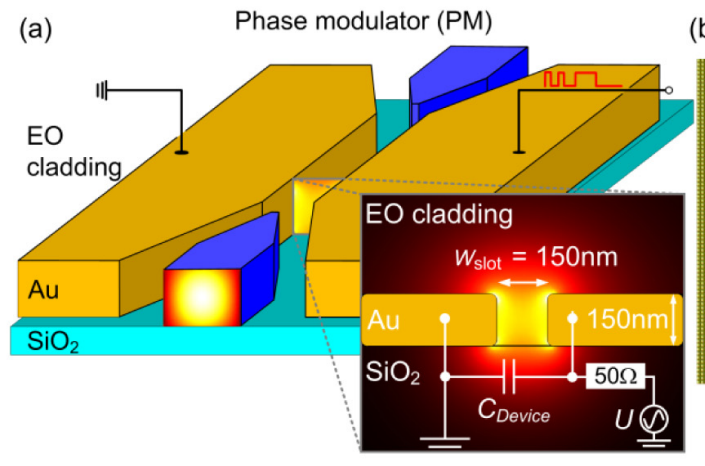

(b)
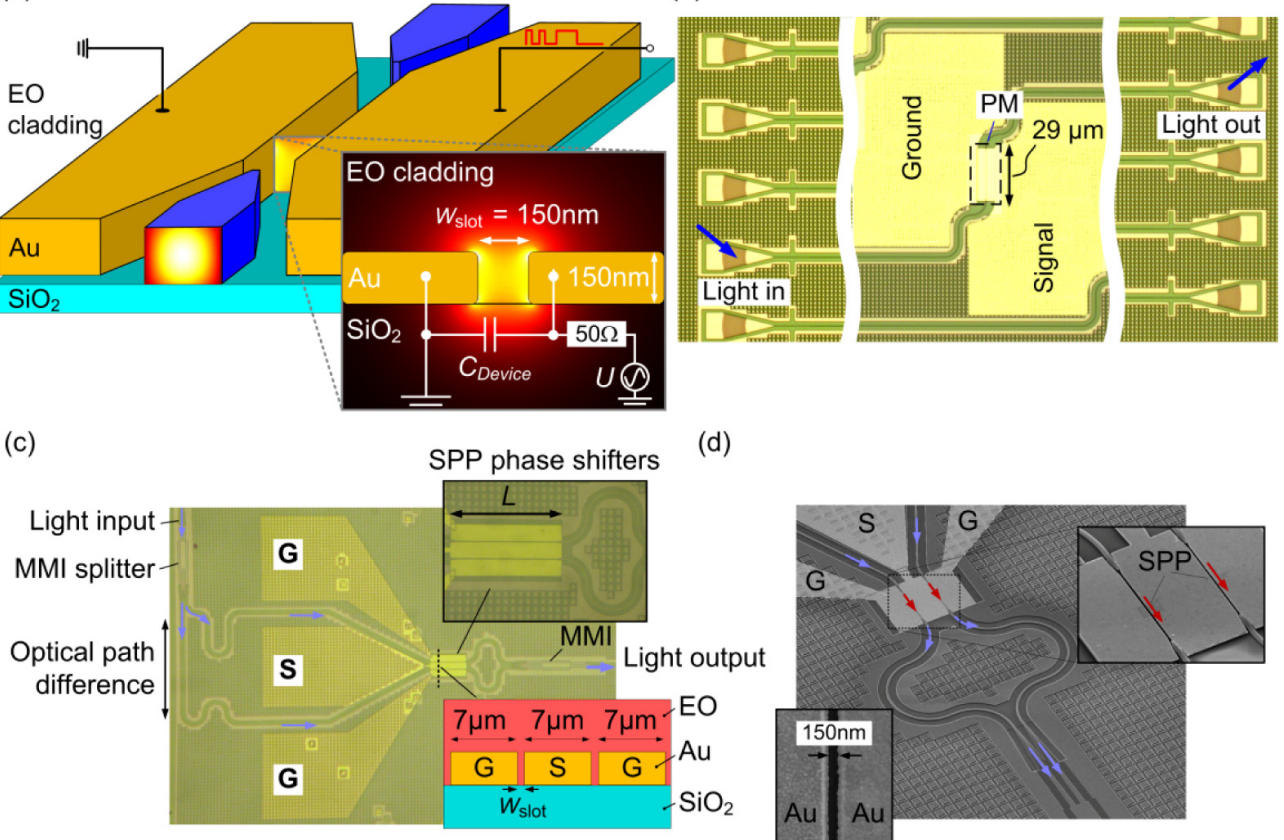

(d)

Fig. 1. Plasmonic-organic hybrid (POH) phase modulator (PM) and Mach-Zehnder modulator (MZM), fabricated on the silicon-on-insulator (SOI) platform. (a) Schematic of the POH PM comprising a metallic slot waveguide filled and clad with an electro-optic (EO) material. The photonic mode of a silicon nanowire waveguide (blue) is converted to a gap surface plasmon polariton (SPP) via a tapered silicon waveguide enclosed by a tapered gap plasmon waveguide [23]. The inset shows a cross section of the device with the mode field of the SPP in the gap. In addition, a lumped-element equivalent circuit of the PM is given. The device can be represented by a capacitor $\left(C_{\text {Device }} \approx 1.5 \ldots 3 \mathrm{fF}\right.$, length dependent). (b) Optical microscope photograph of the fabricated POH PM. Light is launched in and out of the silicon waveguide via diffraction grating couplers. The phase of the optical signal is modulated in the plasmonic modulator section having a length of $29 \mu \mathrm{m}$. (c) Optical microscope image of the fabricated MZM. The Mach-Zehnder interferometer (MZI) is fabricated on the silicon-on-insulator (SOI) platform using low-loss photonic MMI couplers for light splitting and combing. An optical path difference is implemented in the MZI, and the operating point is selected by choosing the operating wavelength. For modulation, an optical phase difference between the two arms is induced by the SPP phase shifters [19]. (d) Scanning electron microscope (SEM) picture of the silicon-plasmonic MZM. The modes of the silicon waveguide are coupled to the plasmonic phase PM sections, which modulate the phase of the SPPs.

Both, POH-PM and MZM, are fabricated on the same SOI wafer. First, the silicon PLC is fabricated using standard processes such as $193 \mathrm{~nm}$ DUV lithography and Si dry etching in the framework of ePIXfab. The metallic slots having widths of $\sim 150 \mathrm{~nm}$ are defined on the silicon PLC with electron-beam lithography and a lift-off process with poly-methylmethacrylate (PMMA) resist. The PM is fabricated with a ground-signal configuration, see Fig. 1(a) and 1(b) [19]. For the MZM, two high-speed PS with a common signal electrode are fabricated in ground-signal-ground configuration as depicted in Fig. 1(c) and 1(d). The devices are fabricated on gold because of the low plasmonic losses and the expected long term 
stability. In the case of the POH PMs, the slot is filled with an electro-optic (EO) organic material YLD124/PSLD41(25:75 wt.\%) mixture which has been previously shown to provide a record EO coefficient of $r_{33}=230 \mathrm{pm} / \mathrm{V}$ [24]. In the case of the POH MZM, we employ the EO material SEO100 (Soluxra, LLC) because of its excellent thermal stability up to $85^{\circ} \mathrm{C}$, which is an important requirement for use in real-word communication systems. The electrooptic effect in the EO materials is activated through a poling procedure [24].

\section{Plasmonic phase modulators and Mach-Zehnder modulators}

\subsection{Plasmonic-organic hybrid phase modulator}

We first characterize the electro-optic response of the PM. Light from a tunable laser source (TLS) is coupled in and out of the silicon nanowires using the grating couplers. To characterize the modulator's radio frequency (RF) response, the device is driven with sinusoidal RF signals of various frequencies. The driving RF power is kept constant during the frequency sweep by first performing a power calibration before attaching the RF probe. The spectrum of the phase modulated optical signal $\cos \left(\omega_{0} t+\eta \sin \omega_{\mathrm{m}} t\right)$ is measured with an optical spectrum analyzer, and the modulation index $\eta$ is estimated by the ratio of the power at the carrier wavelength and the power at the first sideband [19]. The measured modulation index as a function of the modulating RF frequency $f_{\mathrm{m}}=\omega_{\mathrm{m}} / 2 \pi$ from $f_{\mathrm{m}}=10 \mathrm{GHz}$ up to $f_{\mathrm{m}}=$ $62 \mathrm{GHz}$ is given in Fig. 2(a) for an RF power of $-10 \mathrm{dBm}\left(U_{\mathrm{pp}}=0.2 \mathrm{~V}\right)$ measured across a 50 $\Omega$ resistor. In the measurements, the lower and the upper limits of the frequency range are posed by the resolution bandwidth of the optical spectrum analyzer and by the maximum frequency (for an RF power of $-10 \mathrm{dBm}$ ) of the vector network analyzer, respectively. The black solid line in Fig. 2(a) gives the mean value of two consecutive measurements, while the error bars describe the difference between the two measurements. By knowing the RF response of the single PM, we estimate the $3 \mathrm{~dB}$ bandwidth to be larger than $60 \mathrm{GHz}$ for a MZM comprising our PM and operating at the quadrature point of the MZM transfer function. The slight drop of the modulation index with increasing frequency is attributed mainly to the RF probe, the frequency dependence of which could not be taken into account during the calibration of the RF power at the coaxial probe input. For clarity, we add the decaying frequency response of the probe as a grey solid line in Fig. 2(a). In the range below $10 \mathrm{GHz}$, a flat frequency response is to be expected because of the small capacitance of the device, which has been demonstrated previously [19]. To demonstrate that the new EO material does not also limit the optical bandwidth of the device, we characterize the optical response of the BPSK modulator by keeping the RF constant at $40 \mathrm{GHz}$, and by sweeping the carrier wavelength across the $70 \mathrm{~nm}$ range around $1550 \mathrm{~nm}$, see Fig. 2(b). The flat optical response of the modulator makes the device also suitable for communication systems utilizing wavelength division multiplexing schemes. For a modulation index of $\eta=0.04$ at a sinusoidal RF modulation signal with $40 \mathrm{GHz}$ and a peak-to-peak amplitude of $U_{\mathrm{pp}}=0.4 \mathrm{~V}$ (open circuit condition), we estimate the value of the electro-optic coefficient to be $r_{33}=116 \mathrm{pm} / \mathrm{V}$ for a voltage-length product $U_{\pi} \times L$ of $0.45 \mathrm{Vmm}$. It has been previously shown that larger electrooptic coefficients and thus a smaller $U_{\pi} \times L$ can be achieved with an optimized poling procedure [24]. 

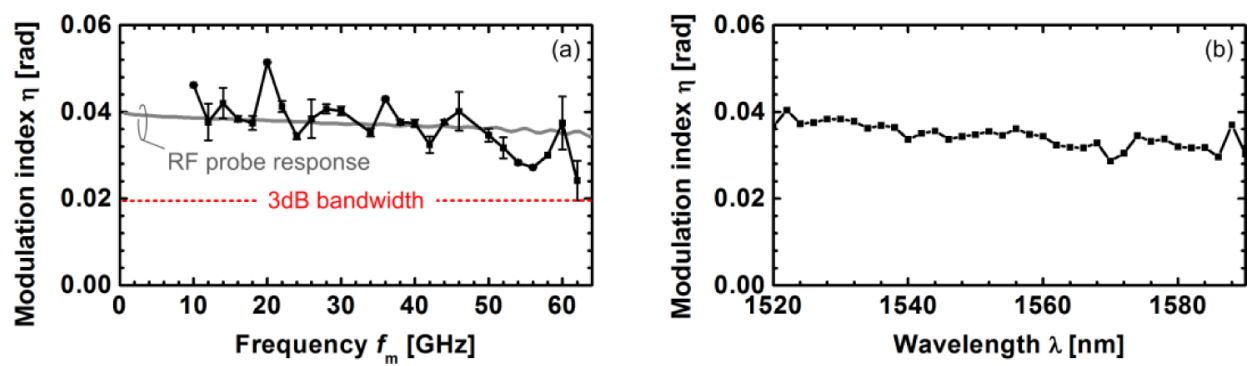

Fig. 2. Electro-optic response of a phase modulator with a length of $29 \mu \mathrm{m}$. (a) Modulation index as a function of the modulating frequency for a sinusoidal RF modulation signal. The RF power amounts to $-10 \mathrm{dBm}$ measured for a $50 \Omega$ terminating resistor. The black solid line gives the mean of two consecutive measurements, and the error bars indicate the difference between the two. For comparison, the grey solid line shows the transfer function of the GS probe from its coaxial input to the GS output as taken from the data sheet. The vertical scale is adjusted to show that for the higher frequencies the drop in modulation index is - to a significant amount-due to the frequency response of the probe. Hypothetical 3dB bandwidth of a MZM comprising the plasmonic PMs is indicated as a red dashed line. (b) Modulation index versus carrier wavelength in a wavelength range of $70 \mathrm{~nm}$ for the modulating $\mathrm{RF} f_{\mathrm{m}}=40 \mathrm{GHz}$.

Next, we encode a BPSK formatted data stream with a bitrate of $30 \mathrm{Gbit} / \mathrm{s}, 35 \mathrm{Gbit} / \mathrm{s}$ and $40 \mathrm{Gbit} / \mathrm{s}$ using the same experimental setup which was used in our previous work [19], see Fig. 3(a). Light with a wavelength of $1550.92 \mathrm{~nm}$ is amplified in an erbium-doped fiber amplifier (EDFA) to a power level of $+10 \mathrm{dBm}$ and launched into the chip. The phase of the SPP is encoded with a $2^{31}-1$ long pseudo-random bit sequence (PRBS) at a voltage swing of $U_{\mathrm{pp}}=3.8 \ldots 4.2 \mathrm{~V}$, measured across a $50 \Omega$ resistor. The resulting BPSK signal is amplified and then detected by an Agilent N4391A Optical Modulation Analyzer (OMA). An equalizer with a filter length of 60 symbols is used. Constellation diagrams with the corresponding error vector magnitudes (EVM) are depicted in Fig. 3(b)-3(d) with 51,200 points per plot. The BER corresponding to the measured EVM of $18 \%$ is below $10^{-10}$ [25], which is 5 orders of magnitude smaller than the previous demonstration [19]. EVMs measured without an equalizer are $\sim 20 \%$ i. e., the BER is less than $10^{-10}$ regardless of the equalizer. The low BER is made possible not by the digital signal processing, but by the large EO coefficient of YLD / PSLD, by the low optical losses in the silicon PLC, and by the comparatively low-loss plasmonic PM itself. Moreover, these low BERs are achieved with significantly lower optical and RF power levels than in the previous demonstration [19]. Representing the PS as a pure capacitor with an estimated capacitance of $\mathrm{C}_{\text {device }}=4.5 \mathrm{fF}$, we calculate an energy consumption of $\left(2 U_{\mathrm{pp}}\right)^{2} \times \mathrm{C}_{\text {device }} / 4 \approx 70 \mathrm{fJ} /$ bit for $40 \mathrm{Gbit} / \mathrm{s}$ operation [26].

(a)
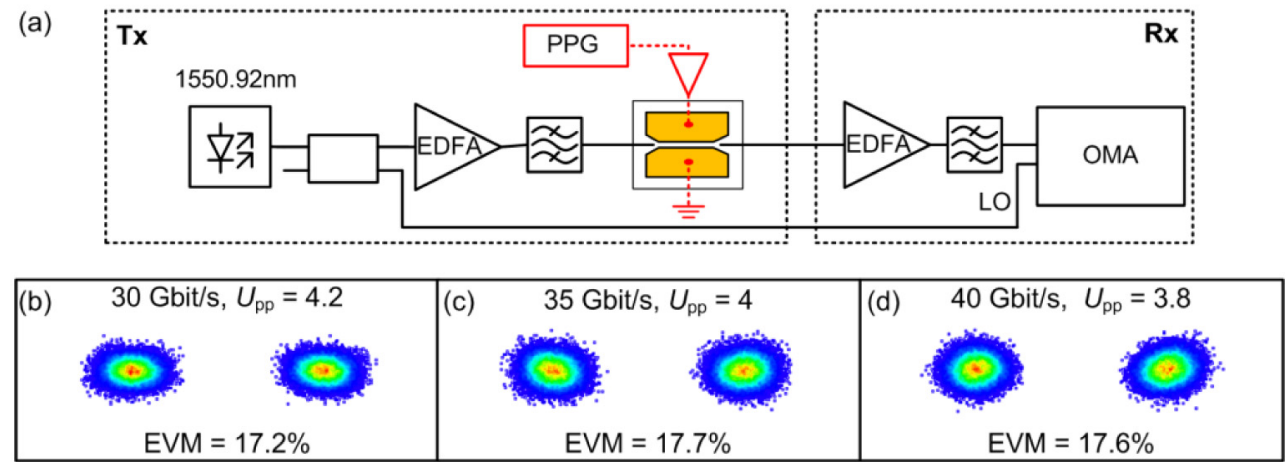

Fig. 3. Experimental setup and constellation diagrams of BPSK data experiments performed with the plasmonic phase modulator. (a) Experimental setup used for generation and detection of the BPSK signals. (b), (c), (d) Constellation diagrams for the bit rates of $30 \mathrm{Gbit} / \mathrm{s}, 35$ Gbit/s, $40 \mathrm{Gbit} / \mathrm{s}$, respectively. Measured EVM are about $18 \%$ corresponding to an BER smaller than $10^{-10}[25]$. 


\subsection{Plasmonic-organic hybrid Mach-Zehnder modulator}

\subsubsection{Static performance}

The POH MZM are first characterized for their static electro-optic response. Light from a tunable laser source is coupled in and out of the silicon nanowire waveguide via diffraction grating couplers. Power transmission spectra for three MZMs with various PS lengths are given in Fig. 4(a). In addition, we display the transmission spectrum of a reference MZI without a PS. It can be seen that the total insertion losses of the MZMs amount to $27 . .34 \mathrm{~dB}$. According to the reference spectrum given in Fig. 4(a), $14 \mathrm{~dB}$ of this loss are caused by the silicon grating couplers, MMIs, and waveguides. The on-chip insertion loss of the PS hence amounts to $13 \ldots 20 \mathrm{~dB}$, depending on the length of the PS. Variations in the slot widths and in the alignments of the metallic tapers can result in a power imbalance and in a change of the optical path length difference in the arms of the MZI, and therefore, in an extinction ratio and a free spectral range (FSR) that are different from the one of the reference MZI, see Fig. 4(a). We analyze the shift of the wavelength corresponding to the minimum transmission as a function of the applied voltage in order to estimate the voltage $U_{\pi}$ which is required for a phase shift of $\pi$. An example of the transmission spectrum for voltage-on and voltage-off states is given in Fig. 4(b) for the MZM with $39 \mu \mathrm{m}$ long phase modulator sections. Measuring the shift $\Delta \lambda_{0}$ for an applied voltage $U_{0}$, we calculate $U_{\pi}=\Delta \lambda_{\mathrm{FSR}} \times U_{0} /\left(2 \Delta \lambda_{0}\right) \approx 30$ $\mathrm{V}$ for the MZM with $39 \mu \mathrm{m}$ long PS, and an $U_{\pi}=37 \mathrm{~V}$ for the MZM with $29 \mu \mathrm{m}$ long PS. The corresponding voltage-length product amounts to $U_{\pi} \times L \approx 1.1 \mathrm{Vmm}-$ one order of magnitude below the values of $10 \mathrm{Vmm}$ as measured for silicon-based carrier-depletion modulators [27]. The in-device electro-optic coefficient $r_{33}$ of the cladding amounts to 70 $\mathrm{pm} / \mathrm{V}$ and is significantly lower than the maximum value of $110 \mathrm{pm} / \mathrm{V}$ specified for bulk SEO100. Thus, $U_{\pi}$ values can be further improved by optimizing the poling procedure of the EO material.

(a)

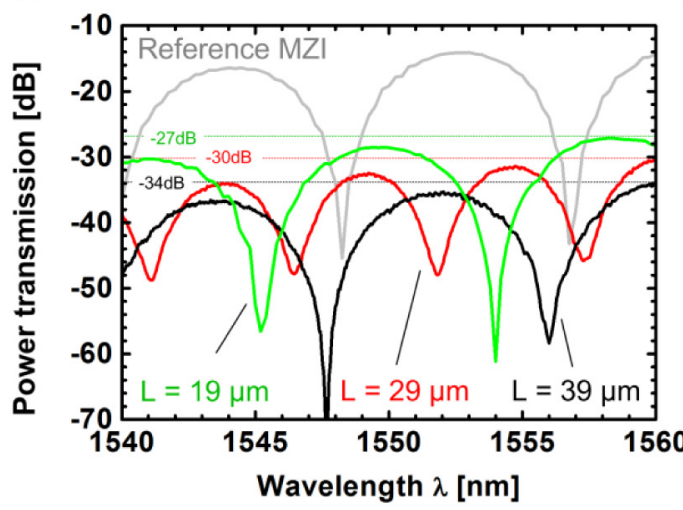

(b)

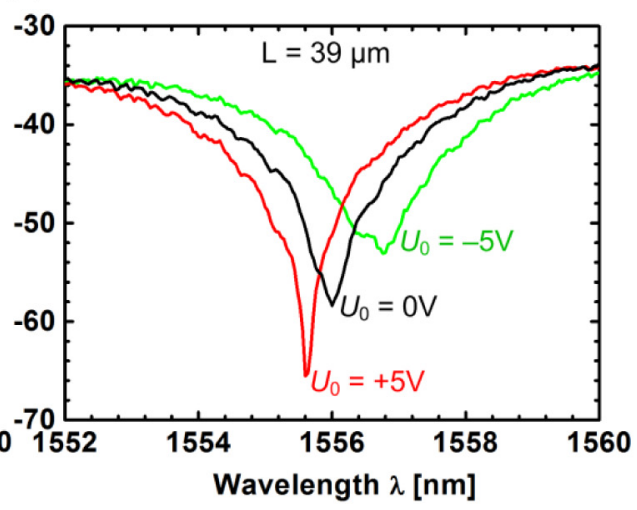

Fig. 4. Static characterization of Mach-Zehnder modulators. (a) Fiber-to-fiber power transmission for MZMs with lengths of $19 \mu \mathrm{m}, 29 \mu \mathrm{m}$ and $39 \mu \mathrm{m}$. In addition, the transmission spectrum of a reference Mach-Zehnder interferometer without PS sections is shown. The plasmonic PS add $13 \mathrm{~dB} \ldots 20 \mathrm{~dB}$ additional optical loss. (b) Transmission spectrum of a $39 \mu \mathrm{m}$ long device for various applied voltages $U_{0}$. Analyzing the shift of the wavelength corresponding to the minimum transmission we estimate the voltage $U_{\pi}$ required for having a phase shift of $\pi$.

\subsubsection{Modulation experiments}

We perform data modulation experiments with our POH MZM using a direct receiver setup as shown in Fig. 5(a). An electrical non-return-to-zero (NRZ) signal with PRBS pattern length of $2^{31}-1$ and with a peak-to-peak voltage swing of $5 \mathrm{~V}$ (measured across a $50 \Omega$ resistor) is fed to 
the modulator via a ground-signal-ground (GSG) RF probe. The operating point for the MZM is defined by selecting the operating wavelength. The MZMs are operated in the quadrature points, i.e., the modulator output intensity changes linearly with the relative phase difference of the two arms. The OOK signal after the MZM is detected with a standard pre-amplified direct receiver comprising a single EDFA, an optical band-pass filter with a bandwidth of 2 $\mathrm{nm}$, a bit-error-ratio tester (BERT), and a digital communication analyzer (DCA).

(a)

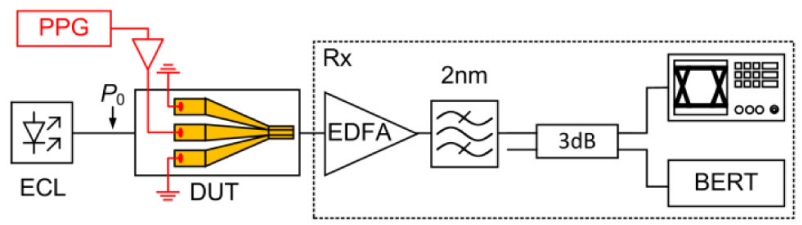

(c) (b)

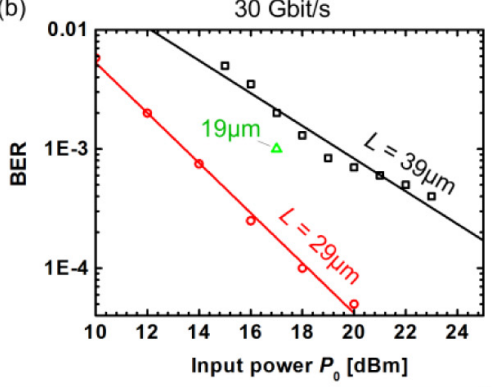

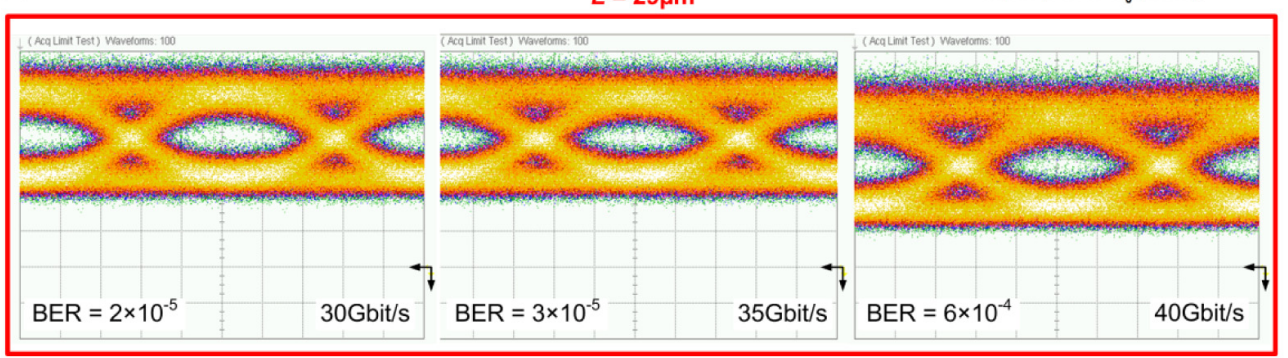

Fig. 5. Modulation experiments with plasmonic silicon-organic MZMs with PS lengths of 19 $\mu \mathrm{m}, 29 \mu \mathrm{m}$ and $39 \mu \mathrm{m}$. (a) Direct receiver setup used for detecting on-off keyed signal after the plasmonic MZMs. (b) Bit error ratios measured for the MZMs with plasmonic phase modulator sections having lengths of $19 \mu \mathrm{m}, 29 \mu \mathrm{m}$ and $39 \mu \mathrm{m}$. To find the optimum phase shifter length, we vary the input power to the modulators and measure the BER. A compromise between the optical loss and the modulation index can be achieved by using a MZM with a PS length of 29 $\mu \mathrm{m}$. (c) Eye diagrams measured at bit rates of $30 \mathrm{Gbit} / \mathrm{s}\left(\mathrm{BER}=2 \times 10^{-5}\right), 35 \mathrm{Gbit} / \mathrm{s}(\mathrm{BER}=3$ $\left.\times 10^{-5}\right)$ and $40 \mathrm{Gbit} / \mathrm{s}\left(\mathrm{BER}=6 \times 10^{-4}\right)$ for a MZM with $29 \mu \mathrm{m}$ long PS sections at an input optical power of $20 \mathrm{dBm}$ and at an operating wavelength of $1556.8 \mathrm{~nm}$. The difference in the DC levels for data rates of $35 \mathrm{Gbit} / \mathrm{s}$ and $40 \mathrm{Gbit} / \mathrm{s}$ is attributed to the thermal drift of the operating point as a consequence of the large optical input power.

We measured the BERs for all three MZMs at a bit rate of $30 \mathrm{Gbit} / \mathrm{s}$ in order to find the optimum length for the phase modulators. During the experiment, the EDFA of the receiver is operated in constant output power mode. The input optical power to the modulator is varied from $+10 \mathrm{dBm}$ to $+23 \mathrm{dBm}$. This varies the input power to the receiver, i.e., the optical signal-to-noise power ratio (OSNR) at the photodiodes. The optimum length of the PS is defined by a compromise between insertion loss and modulation index - making the device too short results in a small optical modulation amplitude, while a too long phase modulator section increases the insertion loss of the device, effectively decreasing the receiver's input power. We find that in our case $\left(U_{\mathrm{pp}}=5 \mathrm{~V}\right.$, SPP propagation losses of $\sim 0.4 \mathrm{~dB} / \mu \mathrm{m}, r_{33}=70$ $\mathrm{pm} / \mathrm{V}$ ) the optimum performance can be achieved with $29 \mu \mathrm{m}$ long phase modulators, see Fig. 5(b). A better BER can be achieved either by increasing the optimum PS length $L$ by improving the slot quality (decreasing optical losses), or by reducing the effective PS length by increasing the electro-optical coefficient and reducing the slot width (increasing the optical modulation amplitude). The eye diagrams measured after the MZM with $29 \mu \mathrm{m}$ long PS sections for bit rates of $30 \mathrm{Gbit} / \mathrm{s}\left(\mathrm{BER}=2 \times 10^{-5}\right), 35 \mathrm{Gbit} / \mathrm{s}\left(\mathrm{BER}=3 \times 10^{-5}\right)$ and $40 \mathrm{Gbit} / \mathrm{s}$ $\left(\mathrm{BER}=6 \times 10^{-4}\right)$ are given in Fig. $5(\mathrm{c})$. These BER are well below the threshold of $4.5 \times 10^{-3}$ 
for hard-decision FEC codes with 7\% overhead [28]. The driving voltages and the optical insertion losses can be further reduced by, first, optimizing the poling procedure and thereby achieving higher electro-optic coefficients, second, reducing the slot size, and third, by using silver instead of gold.

\section{Summary}

We report on error-free generation and detection of $40 \mathrm{Gbit} / \mathrm{s}$ binary phase-shift keying (BPSK) signals using a $29 \mu \mathrm{m}$ plasmonic-organic hybrid (POH) phase modulator with an energy consumption of $70 \mathrm{fJ} / \mathrm{bit}$. Furthermore, we report on on-off keying (OOK) signaling with POH Mach-Zehnder modulators (MZM) at data rates of up to $40 \mathrm{Gbit} / \mathrm{s}$ with low energy consumptions of $75 \ldots 225 \mathrm{fJ} /$ bit. The reported MZM comprise POH phase modulators with lengths of $19 \mu \mathrm{m}, 29 \mu \mathrm{m}$ and $39 \mu \mathrm{m}$. Using the $29 \mu \mathrm{m}$ device, we show OOK signaling at data rates of $30 \mathrm{Gbit} / \mathrm{s}, 35 \mathrm{Gbit} / \mathrm{s}$ and $40 \mathrm{Gbit} / \mathrm{s}$ with the BERs well below the hard-decision FEC threshold. The measured BERs represent the lowest values that have so far been shown with plasmonic modulators at technically relevant data rates. Our findings clearly demonstrate the applicability of plasmonic devices particularly in short-reach optical links.

\section{Acknowledgments}

We acknowledge support from the EU research projects NAVOLCHI, the Center for Functional Nanostructures (CFN), the Karlsruhe School of Optics \& Photonics (KSOP), from the Helmholtz International Research School for Teratronics (HIRST), the German Research Foundation (DFG), Karlsruhe Nano Micro Facility (KNMF), the EU research projects PHOXTROT and BigPipes, from the European Research Council (ERC Starting Grant 'EnTeraPIC', grant no. 280145), and from Alfried Krupp von Bohlen und Halbach Foundation. Silicon waveguides are fabricated by imec in the framework of ePIXfab. We further acknowledge support by Deutsche Forschungsgemeinschaft and Open Access Publishing Fund of Karlsruhe Institute of Technology. 